\title{
Articulatory deficits in Parkinsonian dysarthria: an acoustic analysis
}

\author{
H Ackermann, W Ziegler
}

\begin{abstract}
Twelve patients with idiopathic Parkinson's disease had acoustic speech analysis of sentence utterances to provide information on speech tempo and accuracy of articulation. As a measure of rate of speech the duration of opening-closing movements during articulation was determined from speech wave variables. The intensity of sound emission during articulatory closure as required for stop consonant production, for example, $/ \mathrm{p} /$, $|t|,|k|$, was used as an index of the degree of closure. Speech tempo was not significantly different from normal. The patients, however, had a reduced capacity of completing articulatory occlusion. This was interpreted as reflecting a reduction in movement amplitude of the articulators. Articulatory "undershoot" was not uniform but influenced by linguistic demands in that the closures associated with a stressed syllable were performed at the expense of unstressed ones. Furthermore, switching between opening and closing movements of the articulators in sentence production seemed undisturbed. These results indicate that motor planning of speech differs from arm movement control.
\end{abstract}

The motor deficits of hypokinesia (reduced range of simple limb movements with consequent target undershooting) and bradykinesia (slowness of movements) are characteristic of Parkinson's disease (PD). ${ }^{1}$ In speech, perceptual as well as acoustic signs of hypokinesia in articulation have been widely presumed. ${ }^{2-11}$ Moreover, kinematic analyses have provided direct evidence of reduced and slowed articulatory movements particularly of the lips and the jaw in patients with PD. ${ }^{12-17}$ Studies of limb motor control have shown not only slowness of single movements but also a delay in executing complex motor sequences due to problems in "switching" from one motor programme to another. The impairment in running motor programmes seems to be closely related to the degree of clinical bradykinesia. ${ }^{1}$ Whether this principle applies to speech control as well is not known.

Prosodic features such as linguistic or emotional stress influence speech movements in sentence production. In normal speech the production of stressed syllables is associated with increased articulatory effort. ${ }^{18}$ This raises the question of how far patients with PD are able to produce such extra effort, suggesting that sentence utterances rather than simple repetitive oral movements ought to be used in investigating Parkinsonian dysarthria.

We used acoustic data for the description of hypokinesia and bradykinesia within the speech motor system of patients with PD. Acoustic analysis was based on variables characterising the sound wave emitted during speaking and allows limited inferences about articulatory features. A sentence repetition paradigm was used to approximate most closely the conditions of natural speech to ascertain the effectiveness of certain linguistic constraints, that is, sentence accent, on speech movements in Parkinsonian dysarthria.

\section{Subjects and methods \\ Subjects}

Twelve patients with idiopathic Parkinson's disease (seven men, five women, 47 to 76 years, median 62 years) participated in this study. The clinical data are presented in the table. All patients were on drug therapy; none had positive signs of dementia at psychopathological examination. The range of overall motor impairment at the time of speech evaluation extended from slight akinetic-rigid states to severe disability. Two patients suffered from levodopa induced on/off fluctuations; in both, the speech recordings were obtained during the off state. The control group included 12 "young normals" (seven men, five women, 19 to 35 years, median 29 years) and 12 "elderly normals" (eight men, four women, 42 to 64 years, median 53 years).

\section{Speech examination}

All subjects underwent speech examination in a sound attenuated room. Recordings were made on a Nagra IV S tape recorder with a Sennheiser microphone positioned about $20 \mathrm{~cm}$ in front of the subject. Speech materials included sentences of the form "Ich habe /geCVCe/ gehört" ("I have heard ...") with $\mathrm{C}=/ \mathrm{p} /, / \mathrm{t} /$, $\mid \mathrm{k} /$ and $\mathrm{V}=|\mathrm{i} /,| \mathrm{y} /,|\mathrm{u} /,| \mathrm{a} /$. The formula /geCVCe/ together with the lists of consonants and vowels to substitute for $\mathrm{C}$ and $\mathrm{V}$ enabled the use of 12 target words-for example, "getite", "gepype", "gekuke". These "words" represent regular phonological forms of standard German, so that to a certain extent the sentences fulfil natural speech demands. The 
Table Clinical data of patients with Parkinson's disease

\begin{tabular}{|c|c|c|c|c|c|c|c|c|c|}
\hline \multirow[b]{2}{*}{ Patient } & \multirow[b]{2}{*}{ Age } & \multirow[b]{2}{*}{ Sex } & \multirow{2}{*}{$\begin{array}{l}\text { Duration } \\
\text { of disease } \\
\text { (years) }\end{array}$} & \multicolumn{3}{|c|}{ Motor signs (limbs) } & \multirow[b]{2}{*}{ Depression } & \multirow{2}{*}{$\begin{array}{l}C T \text {-scan } \\
\text { (atrophy) }\end{array}$} & \multirow[b]{2}{*}{ Medication } \\
\hline & & & & akinesia & rigidity & tremor & & & \\
\hline 1 & 47 & $\mathrm{~m}$ & 12 & $(+)$ & - & - & - & - & ld,am \\
\hline 2 & 60 & $\mathrm{~m}$ & 4 & $(+)$ & - & + & - & $(+)$ & ld,ac \\
\hline 3 & 69 & f & 1 & + & $(+)$ & - & - & - & ld \\
\hline 4 & 63 & $\mathrm{~m}$ & 5 & $(+)$ & $(+)$ & - & $(t)$ & - & ld,am \\
\hline 5 & 73 & f & 10 & $(+)$ & $(+)$ & - & - & $(+)$ & ld,am,br \\
\hline 6 & 58 & f & 10 & $(+)$ & $(+)$ & $(+)$ & - & - & ld,br,ac \\
\hline 7 & 57 & $\mathrm{~m}$ & 9 & + & + & $(+)$ & $(+)$ & $(+)$ & ld,br \\
\hline 8 & 71 & $\mathrm{~m}$ & 2 & + & $(+)$ & + & - & - & ac \\
\hline 9 & 66 & $\mathrm{~m}$ & 12 & + & $(+)$ & - & - & - & ld,br \\
\hline 10 & 52 & f & 8 & + & $(+)$ & - & - & - & ld,br \\
\hline 11 & 60 & f & 6 & ++ & + & - & - & - & ld, br,ac \\
\hline 12 & 76 & $\mathrm{~m}$ & 13 & ++ & + & + & $(+)$ & $(+)$ & ld, br \\
\hline
\end{tabular}

$\mathrm{Id}=$ levodopa, $\mathrm{br}=$ bromocriptine, am $=$ amantadine, $\mathrm{ac}=$ anticholinergic drugs $-=$ missing,$(+)=$ slight,$+=$ moderate, $++=$ distinct.

test sentences were read by the examiner in a quasi-randomised order and repeated by the subjects at a suitable rate and loudness level. The entire test, consisting of 12 test sentences, was run twice by all speakers.

\section{Perceptual evaluation}

To obtain perceptual judgments the patients' recordings were evaluated by five experienced speech therapists. The evaluation protocol included seven-point ratings of variables such as a patient's overall articulatory and vocal impairment and his or her speech tempo.

\section{Acoustic evaluation}

Sound pressure level (SPL) contours Speech signals were digitised at a sampling rate of $20 \mathrm{kHz}$ after low-pass filtering at $9 \mathrm{kHz}$ and were fed into a LSI 11/73 for further processing. Sound pressure level (SPL) contours were calculated for each of the 24 sentences. The SPL was determined every $3.2 \mathrm{~ms}$ over a $1 \cdot 2 \cdot 6$ $\mathrm{ms}$ window and the resulting contour was smoothed by a $30 \mathrm{~Hz}$ low-pass filter. Figure 1 provides examples for the test sentence "Ich habe gepipe gehört" as spoken by a normal subject and a patient with PD. During complete closure (as required for the stop consonant sequence $/ \mathrm{b} /, / \mathrm{g} /, / \mathrm{p} /, / \mathrm{p} /, / \mathrm{g} /$ of the test sentence of figure 1 ) intensity is normally

Figure 1 Sound pressure level $(S P L)$ contours of test sentence "Ich habe gepipe gehört" obtained from normal speaker (upper half) and from patient with Parkinson's disease (lower half). Trace at top shows oscillogram of test sentence as spoken by normal subject. Vertical lines mark syllable boundaries, triangle indicates onset of stressed vowel. Above oscillogram part of test sentence "ich habe gepipe gehört" is depicted. Reversed " $e$ " represents phonetic symbol denoting short and centralised esound.
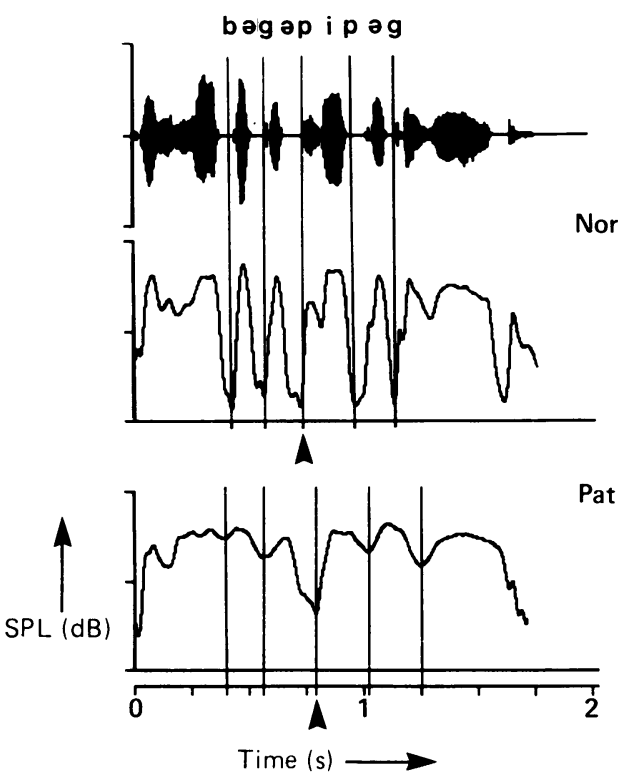

reduced to the level of the tape noise, whereas vocal tract opening for a vowel is associated with an intensity peak. Incomplete closure resulting from reduced extension of mandibular, labial, or lingual movements or from reduced occlusive force may be expected to result in an increase of sound pressure during stop realisation (as seen in the lower half of figure 1) as acoustic energy is still emitted from the mouth. ${ }^{19}$ SPL contours allow calculation of syllable durations and of the amount of sound emission during closure, and moreover reflect dynamic aspects of sound pressure change during closing and opening movements.

Mean syllable duration (MSD) The durations of four syllables of each test utterance were measured: /be/, /ge/, /CV/, /Ce/ (such as $/ \mathrm{be} /, / \mathrm{ge} /, / \mathrm{pi} /, / \mathrm{pe} /$ in the example of figure 1 ). Syllable duration was determined by measuring the time period of a syllabic peak in the SPL contour. Specifically the duration of a syllable was defined as the time interval between the two points where the SPL contour crossed the 5\% threshold between the SPL maximum corresponding to the syllabic peak and the minima left and right of it. ${ }^{19}$ The four syllable durations of a sentence were averaged and the median value of the 24 test utterances of the subject was calculated. We called this measure the mean syllable duration (MSD). As the syllables used in the computation of MSD consisted of a stop consonant and a vowel each, MSD may be considered here as reflecting the time interval of an opening-closing cycle. By virtue of its definition MSD provides an index of speech tempo which is unaffected by pausing, syllable iteration, or by difficulties in word-finding or speech initiation problems that may also be present in PD. ${ }^{20}$

Intensity during closure (IDC) For the two stop consonants preceding and following the (stressed) target vowel of a test word (for instance the two $/ \mathrm{p} / \mathrm{s}$ in the example of figure 1 ) intensity minima were computed. As mentioned above this variable measures the acoustic quality of stop consonant production and is particularly sensitive to incompleteness of closure (undershooting). Median values of intensity minima were determined over all 24 sentences of a subject for each of the pre- and post-stressed closures, respectively. We called the resulting measure the intensity during closure (IDC). 
Figure 2 Perceptual judgments of five speech pathologists for patients overall impairment of voice (abscissa) and articulation (ordinate) given in mean rating scores.

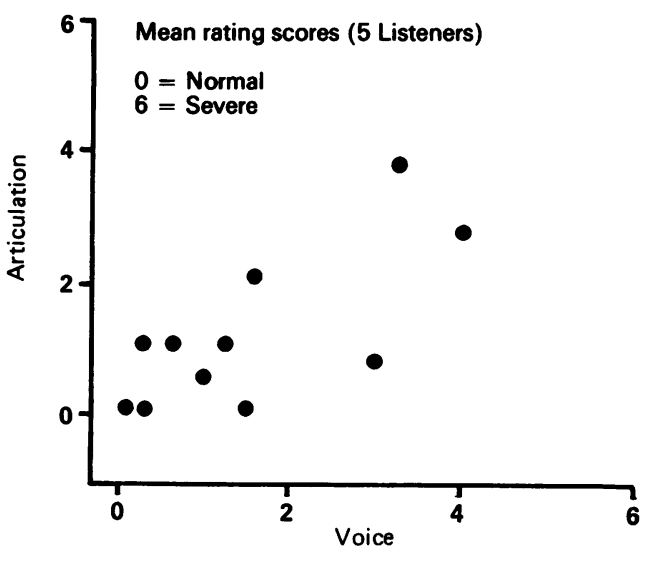

Transition quotient ( $T Q$ ) The period of full opening during vowel production may with some justification be considered a steady state, characterised by temporarily stable acoustic conditions, in contrast with the intervals of opening and closing where acoustic conditions are subject to fast changes. The relative durations of these periods wichin a consonantvowel-consonant cycle contain dynamic information on the underlying movements. In particular, the relative duration of the steady state interval may be considered an index of the time period when the switch between the opening and closing gestures occurs. We considered the period between the $95 \%$ thresholds of the rising and falling SPL contour the steady-state portion and the remainder of the full syllabic cycle (the two $5 \%-95 \%$ slopes) as the movement portion. A transition quotient (TQ) was computed by dividing the movement portion of a syllable by the full syllable duration. This quantity was determined for the target CV syllable in each sentence and again the median value over all utterances of a subject was used to characterise his or her performance.

\section{Results}

Perceptual evaluation Figure 2 presents the results of perceptual judgments of the patients' overall impairment of voice (abscissa) and articulation (ordinate). The data reveal that most cases were judged to be of mild to moderate severity. One patient (number 1) was

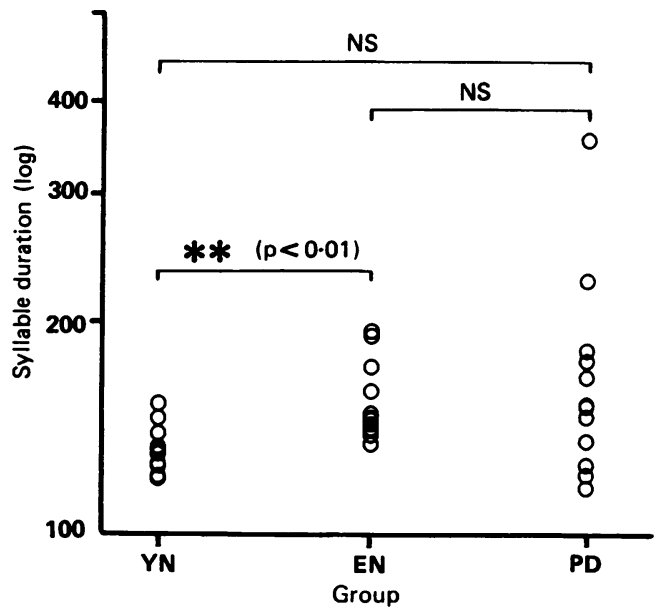

considered normal and three were more severely dysarthric (numbers 10, 11, 12). Impairment of vocal variables-for example, vocal intensity, pitch, and intonation-was judged more severe than articulatory deficits, although the difference was only marginally significant (Wilcoxon, $\mathrm{p}<0.05$ ). Considering articulation alone, three patients were judged as undisturbed and two further patients obtained mean scores lower than 1 .

Speech tempo The mean syllable durations obtained in the two normal groups and in the patients with PD respectively are shown in figure 3. Nonparametric testing (MannWhitney) revealed that the young normal group spoke significantly faster than the elderly normal group ( $p<0.01$ ). No significant differences were obtained between the patients with PD, however, and either the young normals or the elderly normals $(p>0 \cdot 1)$. The two clinically most impaired patients (numbers 11 and 12 in the table) presented with increased MSD, whereas three further patients were slightly faster than the elderly normals, showing mean syllable durations within the lower range of the young normals. In accordance with these measurements, perceptual evaluation indicated slowed speech in the former two and accelerated speech in the latter three patients. No correlation between degree of severity as judged by perceptual evaluation and mean syllable duration could be detected (Spearman, $\mathrm{r}=0 \cdot 26, \mathrm{p}>0 \cdot 1$ ).

Articulatory precision Unlike mean syllable duration, intensity during closure (IDC) was substantially disturbed in the patients as a group. Figure 4 shows that the IDC values of the post-stressed consonant were significantly increased in the patients compared with either of the two normal groups. A significant correlation between perceived severity of overall articulatory impairment and IDC was found (Spearman, $r=0.64, p<0.05$ ). Whereas the patients who were judged as having little or no

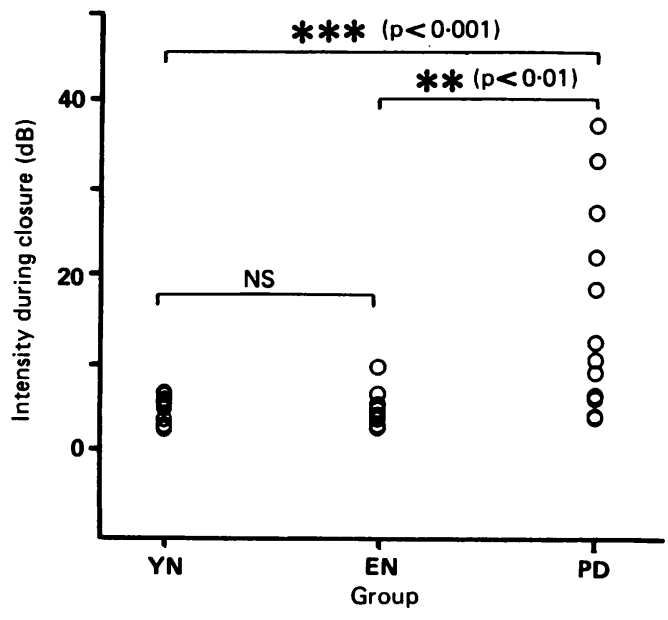

Figure 4 Intensity during closure (IDC)-that is, minimal sound pressure level (SPL) during stop consonant production-in young normals ( $Y N$ ), elderly normals (EN), and patients with Parkinson's disease (PD). For each subject median value determined from post-stressed closures over all 24 sentences is depicted, reflecting articulatory accuracy of stop consonant production. 
Figure 5 Difference of intensity during closure (IDC) between stops preceding and following stressed target vowel in young normals ( $Y N$ ), young normals
elderly normals $(E N)$, and patients with Parkinson's disease (PD). Median values over all 24 sentences of each subject are depicted.

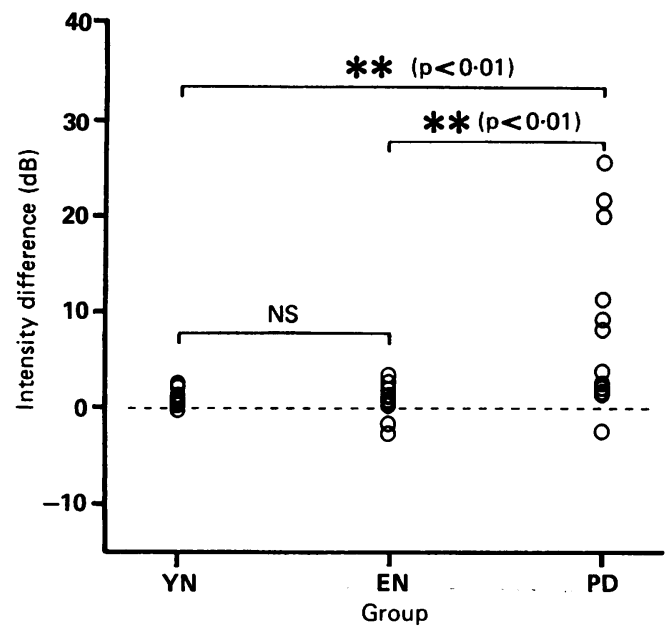

articulatory impairment assumed IDC values within the normal range, and considerably increased values were obtained in the moderate and severe cases.

Production of sentence accent Figure 5 depicts the IDC difference between the stop consonant of the post-stressed and that of the stressed syllable in the three experimental groups. In almost all normal speakers the IDC values were higher in the post-stressed than in the stressed position. This corresponds to expectations as the realisation of stress is generally assumed to be associated with an increase in muscular activity. ${ }^{21}$ In the patients with PD the IDC differences were positive, suggesting their sentence productions followed a normal accent pattern. The resulting difference values were significantly enhanced in the patients relative to the normal subjects, which may mean that their efforts to produce sentence accent were at the expense of an overproportionate-deterioration of the post-stressed stop consonant.

Transition quotient Computation of the transition quotient (TQ) revealed no significant differences between patients with PD and normal speakers (figure 6). There was a tendency to enhanced movement times both in elderly normals and patients compared with the younger normals. These differences, however, were not significant. To the extent that the complement of TQ may be considered an index of the faculty of switching between two movement directions (opening and closing) our results show that the patients examined here had no particular problems in this respect.

Figure 6 Transition quotients (TQ) - that is, movement portion of syllable divided by full syllable duration-in young normals $(Y N)$, elderly normals (EN), and patients with Parkinson's disease (PD). Median values over all utterances of each subject are given.

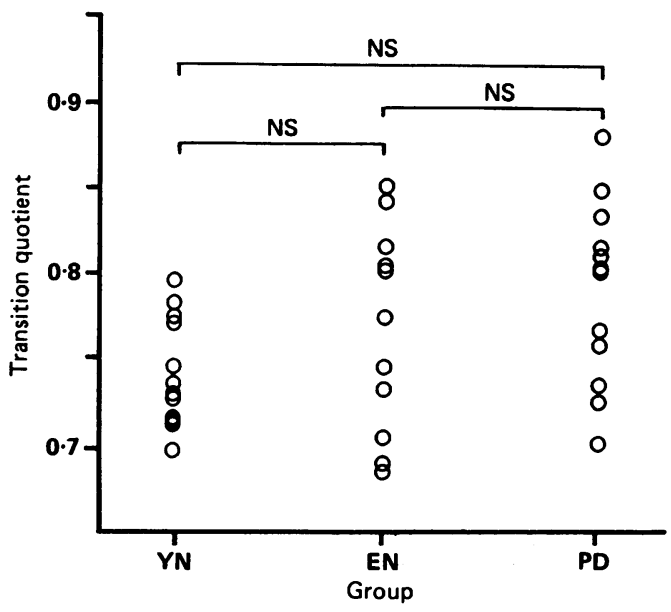

Discussion

Speech motor deficits can be described on the basis of perceptual, acoustic, or physiological (EMG, dynamic, or kinematic) measures. Perceptual assessment of dysarthria may cover those aspects of a patient's speech impairment that are most relevant from the viewpoint of his or her communication needs. Descriptions based on auditory parameters, however, are generally not reliable ${ }^{22}$ and are not easily interpretable in terms of underlying pathological mechanisms. $^{23}$ In contrast, physiological assessment techniques such as the $x$ ray microbeam, ${ }^{12-14}$ strain-gauge measurements, ${ }^{15}{ }^{16}$ or electromagnetic articulography, ${ }^{24}$ yield direct observations of speech motor patterns. Yet data obtained by these techniques are inherently difficult to interpret as between subject variation is considerable. ${ }^{25}$ As an example, the data reported by Forrest $e t a^{17}$ may be mentioned. Their measurements of jaw opening amplitudes showed that their normal subjects varied between values characteristic of their most severe examples of Parkinsonism and values two times as high as the maximum obtained in their patients, although jaw movement amplitudes were among the measures that showed the highest group differences between the patients and the normal group in their study. Not unexpectedly therefore there is a notoriously low correlation between movement data and perceived degrees of dysarthric impairment. ${ }^{26}$ Moreover, the recording of speech movements by physiological techniques usually requires a high amount of cooperation of the subject if reliable data are to be produced, a precondition that often cannot be met in many subjects with neurological illness. Further, these techniques potentially disturb natural speech production, which imposes particular limitations on the analysis of more complex utterances like sentences. This may be one reason why parametric studies of articulation in Parkinsonism have so far focused on elementary speech tasks-for example, single word production or rapid syllable repetition (diadochokinesis). Although diadochokinesis seems to predict the overall adequacy of speech in dysarthria fairly well, ${ }^{2}$ the syllable repetition tasks commonly employed in the measurement of diadochokinetic rates have the disadvantage of differing from natural speech in terms of the underlying motor organisation.

A way to circumvent the limitations of physiological assessment techniques and, at the same time, obtain objective and quantitative data, is provided by acoustic analyses of the speech wave. Although a one-to-one correspondence between acoustic measures of speech sounds and the corresponding speech movements does not exist, certain inferences concerning disturbed motor patterns may nevertheless be drawn from acoustic data if speech tasks are appropriately designed. Most importantly, acoustically verified deviations from a given target may be considered to reflect relevant aspects of disturbed speech rather than functionally irrelevant idiosyncracies of articulation. 
In accordance with earlier studies ${ }^{5-7}$ our patients displayed on average slightly more pronounced phonatory than articulatory disturbances, as far as clinical-perceptual ratings are concerned. Logemann and Fisher suggested a progressive involvement of speech organs in PD, beginning at the laryngeal level and proceeding in the oral direction. ${ }^{7}$ An alternative explanation would be that the laryngeal apparatus is more vulnerable to the pathophysiological processes underlying $\mathrm{PD}$, meaning that even very mild motor disturbances have clearly audible consequences. Finally, impairment of respiratory support may contribute to phonatory problems in patients with PD.

Our main acoustic finding was a relative preservation of speech tempo and, at the same time, a reduction of articulatory precision in stop consonant production. A normal or even increased rate of speech has often been reported in $\mathbf{P D}^{27}{ }^{28}$ and is considered specific for this disorder by some authors. ${ }^{52}$ At first sight the finding of a normal or even increased speech tempo seems to be incompatible with the concept of bradykinesia, which is a well known feature of limb akinesia in PD $^{1}$ and has been found in the speech motor system as well. ${ }^{17}$ Yet, the observation of normal or reduced syllable durations does not necessarily mean that the underlying articulatory movements were of a normal or even increased velocity. The fact that articulatory closure for stop consonant production was obviously incomplete in some of the patients suggests that these patients performed articulatory movements of reduced amplitude. Despite possible bradykinesia they seem to have produced normal syllabic rates at the expense of movement excursion. This is in agreement with the findings of Forrest $e t$ al of consistent reductions in both peak velocity and amplitude of mandibular and labial opening gestures at normal movement times. ${ }^{16} 17$

This trade off between tempo and articulatory precision is made possible by the fact that reduced articulatory precision may still be compatible with the requirements of intelligible speech. In this respect speech is different from other motor acts, like grasping, where a similar trade off cannot be made without compromising the requirements of the motor task. A task more similar to speech would be handwriting, with the typical symptom of micrographia being observable in PD. Micrographia resembles hypokinetic speech in that smaller movement excursions compensate for the inability to execute high velocity strokes. ${ }^{30}$ Thus the results of speech analysis are not incompatible with the concept of bradykinesia as outlined for limb motor control, emphasising the slowing down of individual movements due to inadequate "energising" and "scaling" of initial agonist muscle activity in relation to functional demands. ${ }^{31} 32$

The tempo-precision trade off is well known from normal speech, where an increase in speech rate is usually achieved by a reduction of movement amplitudes rather than by speeding up the movements themselves. ${ }^{33}$ In dysarthria the maintenance of a normal rate at the expense of articulatory precision is far from universal. On the contrary, most dysarthric syndromes are associated with slowed speech and, at the same time, undershooting articulation. ${ }^{5}$ The faculty of maintaining a normal syllabic rate therefore seems characteristic of the Parkinsonian type of dysarthria.

Yet, following our data and also our earlier observations, the syndrome of Parkinsonian dysarthria is by no means homogeneous with respect to speech rate. Seguier et al observed slowed speech in $5 \%$ of their patients. ${ }^{29}$ In our sample, two patients had increased syllable durations, though the observed increase must be considered fairly mild when compared to spastic dysarthria of similar severity. ${ }^{34}$ Both subgroups with either increased or decreased MSD presented with hypometria in terms of incompleteness of closure. Possibly the two different types reflect consecutive stages in the development of Parkinsonian dysarthria or different degrees of impairment, respectively. According to the clinical impressions and perceptual judgments of experts the two patients with increased MSD were most severely affected, with severe phonatory deficits in addition to their articulation problems. At this severity the maintenance of a normal syllabic rate at the expense of articulatory precision might have led to completely unintelligible articulation, causing the patients to slow their speech. An alternative explanation would be that a critically reduced velocity of laryngeal abductions and adductions forced the patients to lower their syllabic rate.

Articulatory imprecision was not uniformly distributed among the stop consonants of a sentence but rather varied systematically with the sentence accent. Comparison of closures of the stressed and the post-stressed syllable of a sentence showed normal or almost normal performance in the stressed position but an overproportionately reduced realisation in the post-accent position. In other words, hypokinesia affects successive articulatory gestures of an utterance to different degrees. Obviously, the patients with PD were forced by the linguistic status of the stressed CV-syllable to raise more "effort" in the closing movement. Thus inappropriate "energising" of agonist muscle activity and inadequate "scaling" of agonist burst size to task requirements ${ }^{3132}$ are not universal features of Parkinsonian motor deficits. With respect to complex speech motor patterns patients with PD seem to be capable of appropriate "energising" and "scaling" of one elementary movement at the expense of others, dependent upon linguistic demands.

Clinical and experimental data indicate that patients with PD have difficulties in "running" a sequence of motor programmes linked together within a complex motor plan-that is, in "switching" from one motor programme to the next one. ${ }^{135}$ For instance, when performing a sequence of two simple movements patients show a prolongation of the interval between the two elementary actions. Thus in complex movements there is an extra delay in addition to the increased slowness of the individual 
movements. ${ }^{36} 37$ Similarly, when drawing triangles and squares the pauses at the angles are increased. ${ }^{38}$ Connor et al showed that isolated syllables and syllables produced within repetition trains do not differ significantly in terms of acoustic variables. ${ }^{10}$ As repetitive syllable production should have greater demands on motor planning than isolated syllables these findings seem contrary to planning deficits in speech motor control of patients with PD. Syllable repetitions, however, may differ from connected speech insofar as an iteration of the motor programme or highly automatised processes may take over. Our analysis of the open close transitions in sentence production showed no deviations for the utterances of the patients as transition quotients were within normal limits. One might consider this result as being in contrast with the observations of Benecke $e t a l^{36}{ }^{37}$ and Berardelli et $a l^{38}$ In these paradigms, however, the single movements are obviously governed by independent motor programmes. ${ }^{39}$ In contrast, there is some linguistic evidence that complex articulation patterns underlying sentence utterances are under the control of a single "articulatory plan" structured by suprasegmental-that is, prosodic-features. ${ }^{40}$ Therefore, the concatenation of elementary articulations might not be afflicted by the pathophysiological mechanisms outlined by Benecke et $a l^{3637}$ and Berardelli et al. $^{38}$

All acoustic work was carried out by the Neurophonetics Group at the Max Planck Institute for Psychiatry, Munich, under the guidance of W Ziegler. We thank E Hartmann, P Hoole, and I Wiesner for their cooperation. Most of the patients were kindly referred to us by $W$ Hertel, Department of Neurology, Klinikum Großhadern, University (LMU) of Munich. This work was supported by a BMFT grant.

1 Marsden CD. Slowness of movement in Parkinson's disease. Movement Dis 1989;4:S26-S37 (suppl 1).

2 Canter GJ. Speech characteristics of patients with Parkinson's disease. III. Articulation, diadochokinesis, and overall speech adequacy. J Speech Hear Dis 1965;30: 217-24.

3 Darley FL, Aronson AE, Brown JR. Differential diagnostic patterns of dysarthria. J Speech Hear Res 1969;12:246-69.

4 Darley FL, Aronson AE, Brown JR. Clusters of deviant speech dimensions in the dysarthrias. I Speech Hear Res 1969;12:462-96.

5 Darley FL, Aronson AE, Brown JR. Motor speech disorders. London: Saunders, 1975.

6 Logemann JA, Fisher HB, Boshes B, Blonsky ER. Frequency and cooccurrence of vocal tract dysfunctions in the speech of a large sample of Parkinson patients. $J$ Speech the speech of a large samp
Hear Dis 1978;43:47-57.

7 Logemann JA, Fisher HB. Vocal tract control in Parkinson's disease: phonetic feature analysis of misarticulation. $J$ disease: phonetic feature analysi

8 Ludlow CL, Bassich CJ. Relationships between perceptual ratings and acoustic measures of hypokinetic speech. In: McNeil MR, Rosenbek JC, Aronson AE, eds. The dysarthrias: physiology, acoustics, perception, management. San Diego: College Hill, 1984:163-95.

9 Ludlow CL, Bassich CJ. The results of acoustic and perceptual assessment of two types of dysarthria. In: McNeil MR, Rosenbek JC, Aronson AE, eds. The dysarthrias: physiology, acoustics, perception, management. San Diego: College Hill, 1984:121-53.

10 Connor NP, Ludlow CL, Schulz GM. Stop consonant production in isolated and repeated syllables in Parkinson's disease. Neuropsychologia 1989;27:829-38.

11 Weismer G. Articulatory characteristics of parkinsonian dysarthria: segmental and phrase-level timing, spirantiza- tion, and glottal-supraglottal coordination. In: McNeil MR, Rosenbek JC, Aronson AE, eds. The dysarthrias: physiology, acoustics, perception, management. San Diego: College Hill, 1984:101-30.

12 Hirose H, Kiritani S, Ushijima T, Yoshioka H, Sawashima $M$. Patterns of dysarthric movements in patients with Parkinsonism. Folia phoniat 1981;33:204-15.

13 Hirose H, Kiritani S, Sawashima M. Velocity of articulatory movements in normal and dysarthric subjects. Folia phoniat 1982;34:210-5.

14 Hirose H. Pathophysiology of motor speech disorders (dysarthria). Folia phoniat 1986;38:61-88.

15 Hunker CJ, Abbs JH, Barlow SM. The relationship between parkinsonian rigidity and hypokinesia in the orofacial parkinsonian rigidity and hypokinesia in the orofacial
system: a quantitative analysis. Neurology 1982;32: system:

16 Connor NP, Abbs JH, Cole KJ, Gracco VL. Parkinsonian deficits in serial multiarticulate movements for speech. Brain 1989;112:997-1009.

17 Forrest K, Weismer G, Turner GS. Kinematic, acoustic, and perceptual analyses of connected speech produced by Parkinsonian and normal geriatric adults. J Acoust Soc Am 1989;85:2608-22.

18 Sussman HM, MacNeilage PF. Motor unit correlates of stress: preliminary observations. J Acoust Soc Am 1978; 64:338-40.

19 Ziegler W, von Cramon D. Spastic dysarthria after acquired brain injury: an acoustic study. Brit J Dis Comm 1986; 21:173-87.

20 Ackermann H, Ziegler W. Die Dysarthrophonie des Parkinson-Syndroms. Fortschr Neurol Psychiat 1989;57:149-60.

21 Ostry DJ, Keller E, Parush A. Similarities in the control of the speech articulators and the limbs: kinematics of tongue dorsum movement in speech. J Exp Psychol Human Percept Perform 1983;9:622-36.

22 Zyski BJ, Weisiger BE. Identification of dysarthria types based on perceptual analysis. J Commun Disord 1987; 20:367-78.

23 Kent $R$, Netsell $R$. A case study of an ataxic dysarthric: cineradiographic and spectrographic observations. $J$ Speech Hear Dis 1975;40:115-34.

24 Schönle PW, Gräbe K, Wenig P, Höhne J, Schrader J, Conrad B. Electromagnetic articulography: use of alternating magnetic fields for tracking movements of multiple points inside and outside the vocal tract. Brain Lang points inside and

25 Forrest K, Weismer G, Adams S. Statistical comparison of movement amplitudes from groupings of normal geriatric movement amplitudes from groupings of nor
speakers. J Speech Hear Res 1990;33:386-9.

26 Caligiuri $M$. The influence of speaking rate on articulatory hypokinesia in parkinsonian dysarthria. Brain Lang 1989; 36:493-502.

27 Alajouanine T, Sabouraud O, Grémy F. Etude oscillographique de la parole dans la maladie de Parkinson: éffets précoces de la coagulation pallidale. Rev Franc Etudes Clin Biol 1964;9:411-9.

28 Ludlow CL, Connor NP, Bassich CJ. Speech timing in Parkinson's and Huntington's disease. Brain Lang 1987; 32:195-214.

29 Seguier N, Spira A, Dordain M, Lazar P, Chévrie-Muller C. Etude de les relations entre les troubles de la parole et les autres manifestations cliniques dans la maladie de Parkinson. Folia phoniat 1974;26:108-26.

30 Sanes JN, Evarts EV. Psychomotor performance in Parkinson's disease. In: Delwaide PJ, Agnoli A, eds. Clinical neurophysiology in parkinsonism. Amsterdam: Elsevier, neurophysiology

31 Hallett $M$, Khoshbin S. A physiological mechanism of bradykinesia. Brain 1980;103:301-14.

32 Berardelli A, Dick JPR, Rothwell JC, Day BL, Marsden CD. Scaling of the size of the first agonist EMG burst during rapid wrist movements in patients with Parkinson's disease. J Neurol Neurosurg Psychiat 1986;49: 1273-9.

33 Kent $\mathrm{RD}$. The segmental organization of speech. In: McNeilage PF, ed. The production of speech. New York: Springer, 1983.

34 Ziegler W, Hartmann E, Hoole P, von Cramon D. Akustische Dimensionen dysarthrischer Störungsmerkmale. München: GSF, 1990.

35 Marsden CD. The mysterious motor function of the basal ganglia. Neurology 1982;32:514-39.

36 Benecke R, Rothwell JC, Dick JPR, Day BL, Marsden CD. Disturbance of sequential movements in patients with Parkinson's disease. Brain 1987;110:361-79.

37 Benecke R, Rothwell JC, Dick JPR, Day BL, Marsden CD. Simple and complex movements off and on treatment in patients with Parkinson's disease. J Neurol Neurosurg Psychiat 1987;50:296-303.

38 Berardelli A, Accornero N, Argenta M, Meco G, Manfredi $M$. Fast complex arm movements in Parkinson's disease. $J$ Neurol Neurosurg Psychiat 1986;49:1146-9.

39 Benecke R, Rothwell JC, Day BL, Dick JPR, Marsden CD. Motor strategies involved in the performance of sequential movements. Exp Brain Res 1986;63:585-95. 40 Levelt WJM. Speaking. From intention to articulation. 